\title{
The effect of interlayer gap width on burr formation in drilling of aluminium-aluminium aerospace stacks
}

\author{
Andrea Pardo ${ }^{1} \cdot$ Akos Cseke $^{1} \cdot$ Robert Heinemann $^{1} \cdot$ Robert Whiffen $^{2}$ \\ Received: 10 April 2019/ Accepted: 25 July 2019 / Published online: 8 August 2019 \\ (C) The Author(s) 2019
}

\begin{abstract}
Interlayer burr formation during drilling of stacks is a widespread issue in the aerospace industry. The minimisation of the interlayer burr would contribute to significant time and cost savings, as it would allow for clamping, drilling and fastening to be carried out without any intermediate deburring that requires separating the layers. This paper reports about a notbefore observed phenomenon associated with the relationship between interlayer gap width and burr height when drilling aluminium-aluminium stacks with the presence of sealant at the interface. Initial experiments of the research were conducted to determine the interlayer gap widths in relation to a range of clamping forces, followed by drilling experiments to assess how the interlayer gap width affects the interlayer burr formation process and burr height. Although the presence of an interlayer gap results in larger burrs being formed, it allows upwards-travelling chips to enter the gap and erode away the newly formed burr. Larger interlayer gap widths were found to yield a more pronounced abrasion of the interlayer burrs, often leading to their complete removal; in some cases, this abrasion even resulted in a noticeable rounding of the borehole edges. This phenomenon was found to strongly affect the interlayer borehole quality and, thus, makes this research highly significant to the aerospace industry, where the quality of the borehole at the stack interface is of major interest.
\end{abstract}

Keywords Stack drilling · Interlayer gap · Burr formation · Burr abrasion · Clamping force $\cdot$ Stack interface

\section{Introduction}

The design and assembly of aircraft components rely heavily on the use of fasteners [1]. To ensure that the boreholes for each fastener are aligned with the required accuracy, the components are assembled prior to drilling, with the regions where components overlap commonly being referred to as stacks [2]. The drilling of such stacks, referred to as stack drilling, is therefore characterised by the provision of a borehole through a series of different layers, which can comprise of-but is not limited toaluminium, titanium and carbon fibre reinforced plastic

Robert Heinemann

robert.heinemann@manchester.ac.uk

1 School of Mechanical, Aerospace and Civil Engineering, The University of Manchester, Sackville Street, Manchester, M1 3BB, UK

2 Airbus Operations Limited, Chester Road, Broughton, Chester, CH4 ODR, UK
(CFRP). Recently, the research on stack drilling has started to focus on the interface between layers following the desire of aircraft manufacturers to introduce one-way assembly $[3,4]$. This is a combined manufacturing and assembly technique, whereby component layers are fastened together directly after drilling without any intermediate process that would require separating the layers, thereby considerably reducing assembly time. To achieve this, the borehole interface between adjacent layers must be clear of imperfections that would require reworking. This implies that, for example, drilling burrs created between two adjacent layers - often referred to as interlayer burrs-must be kept below a certain limit.

Various studies have focused on the reduction of interlayer burr formation through the optimisation of process parameters in drilling of aerospace stacks. Zhu et al. [5] investigated the impact of tool geometry on burr formation in dry drilling of aluminium-titanium stacks. They observed that double cone drills produce significantly smaller burrs when compared with drills with multipoint and step geometries. Melkote et al. [6] and Tian et al. [7] 
established relationships between feed rate and burr height and thickness, which is explained by the strong correlation between feed rate and thrust force [8]. The interlayer gap width is believed to strongly influence interlayer burr formation and has thus been the subject of extensive research. Jie [9], using an analytical model and experiments, found that applying a pre-load clamping force in the proximity of the drilling site when drilling aluminiumtitanium stacks reduces the interlayer gap width, thereby resulting in smaller interlayer burrs. This observation is in agreement with the work conducted by Li et al. [10] and Yin et al. [11], who focussed on aluminium-aluminium stacks. Gao et al. [12] however point out that increasing the preload clamping force beyond a certain threshold does not lead to a further reduction in the interlayer gap. Melkote et al. [6], who assessed the effect of various clamping methods on interlayer burr formation in drilling of aluminiumaluminium stacks, recorded an increase in interlayer burr height with an increase in the distance between drilling site and clamping location.

The review of the literature suggests that research on drilling of stacked materials with the view on interlayer burr formation exhibits two major shortcomings. Firstly, the actual width of the interlayer gap throughout the drilling cycle was not directly determined, thereby making it difficult to correlate a particular gap width to a certain burr height. Because of the setups used in previous research, which in many cases involved large plates as workpieces, the established relationships between gap width and burr size are specific to the particular setup rather than generic and universally applicable. Secondly, the stacks were drilled with the two layers in direct contact, and no publication was found where prior to drilling an interlayer medium was applied, such as sealants that are commonly used in wingbox assemblies to prevent fuel leakage. However, in order to achieve one-way assembly, the separation of the two layers between drilling the holes and inserting the fasteners needs to be avoided, which means that the sealant has to be applied at the stack interface prior to clamping and drilling. The presence of this sealant film most likely will affect the width of the interlayer gap, resulting, as a consequence, in the possibility that it influences burr formation.

To address these shortcomings, this paper establishes a relationship between interlayer gap width and burr height when drilling aluminium-aluminium stacks with the presence of sealant at the interface. To achieve this, initially, the correlation between the interlayer gap width and clamping load applied was determined and, subsequently, the effect of the interlayer gap on interlayer burr formation was investigated.

\section{Experimental methodology}

\subsection{Experimental setup}

The experiments were carried out on stacks comprising two layers of aluminium 7010-T651, each with a thickness of $10 \mathrm{~mm}$. The material was supplied in $50 \mathrm{~mm}$ x $50 \mathrm{~mm}$ coupons in shot-peened and coated condition. Figure 1 illustrates the composition of the stack, the definition of the interlayer gap width as well as the location of the exit and entry interlayer burrs. Although the use of large plates would have been more representative of aerospace assembly operations, it would have resulted in a significant loss of control over the actual interlayer gap, not only at different hole locations, which would vary substantially along the length of the component, but also throughout each individual drilling cycle. The decision to conduct

Fig. 1 Stack composition

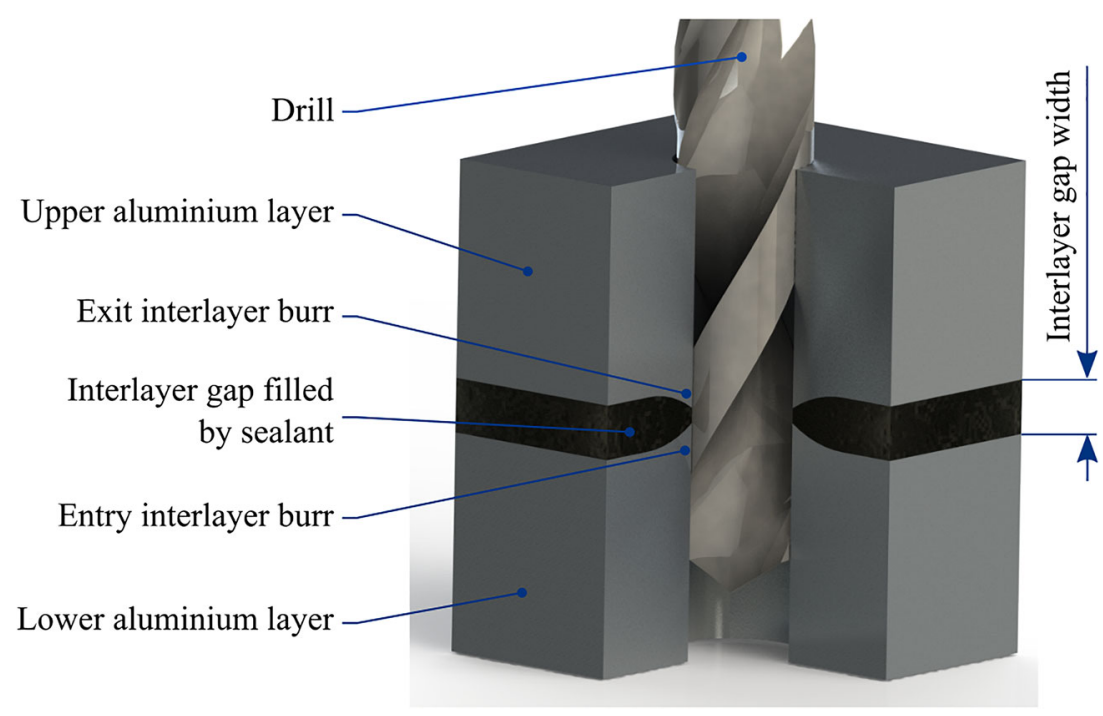


the experiments using the small coupons was thus based on the intention to ensure that the pre-set gap width was maintained throughout the drilling cycle and for all holes drilled. Calculations revealed that the deflection of the stack at the location of the borehole was in the order of $2-3 \mu \mathrm{m}$, based on a thrust force of $650 \mathrm{~N}$, which was determined experimentally. The change in gap width as a result of this deflection was considered negligible.

Despite the fact that this setup is an artificial scenario, the fundamental information gathered with regards to the relation between interlayer gap width and burr size can be applied to the drilling of larger components, where the interlayer gap is subject to change during a drilling cycle, the more the further the hole is located away from the clamping point, as observed by Melkote et al. [6].

The coupons were clamped using a custom-made vice, shown in Fig. 2, which was mounted on top of a twocomponent piezo-electric dynamometer (Kistler 9271A). The bottom layer of each stack rested on two $5 \mathrm{~mm}$ wide shoulders, one on either side of the layer. The vice comprises a rest, bolted on top of the dynamometer, and a frame, which contains a hole through which the drill can access the stack. The rest and frame were connected using two M8 screws. A further recess was machined into the frame to accommodate a pressure ring, which was included to mimic the so-called nose piece used in drilling machines commonly found in the aerospace industry. Two load cells (Omega LCWD-2K) using a strain indicator (VPG Strain indicator P3) were used to determine the axial clamping load applied to the stack through the pressure ring when tightening the M8 screws. The thrust force and torque signals recorded by the dynamometer were fed to a Kistler charge amplifier (type 5070), which was in turn connected through a data acquisition system (Kistler 5697) to a PC running DynoWare. Both thrust force and torque were recorded with a sampling rate of $1 \mathrm{kHz}$.

The cutting experiments were carried out on a three-axis vertical machining centre (Takisawa MAC-V3, equipped with a Semer Anlagen Technik MQL system). The drilling
Table 1 Experimental parameters

Interlayer gap

$0 ; 0.1 \mathrm{~mm} 0.2 \mathrm{~mm}$;

$0.3 \mathrm{~mm} ; 0.4 \mathrm{~mm}$

\begin{tabular}{ll}
\hline Cutting speed & $55 \mathrm{~m} / \mathrm{min}$ \\
Spindle speed & $1379 \mathrm{rpm}$ \\
Feed rate & $0.039 \mathrm{~mm} / \mathrm{rev}$ \\
Feed velocity & $54 \mathrm{~mm} / \mathrm{min}$ \\
Coolant & Accu-Lube LB-4000 \\
Coolant flow rate & $11 / \mathrm{h}$ \\
Pressure ring inner diameter & $36.9 \mathrm{~mm}$ \\
Pressure ring outer diameter & $46.9 \mathrm{~mm}$ \\
Clamping load & $500 \mathrm{~N}$ \\
\hline
\end{tabular}

tools used were two-fluted twist drills manufactured by ProCorp/Sandvik with a diameter of $12.7 \mathrm{~mm}$ (ST00070028). They exhibited a $40^{\circ}$ helix angle, polished chip flutes and four-facet point geometry with additional notches, which resulted in the chisel edge being reduced to a point. The cutting parameters used for the experiments are shown in Table 1.

\subsection{Interlayer gap measurements}

In order to establish the relationship between clamping load and resultant interlayer gap width, a coordinate measuring machine (LK G90C) was used to determine the actual thickness of the stack, i.e. coupons plus sealant (PR1782C12). After having clamped the stack in the vice with a layer of sealant in the interface, the distance between the stack's top and bottom surface was determined. Clamping loads ranging from 100 to $3000 \mathrm{~N}$ were applied, and three different measurements were taken per clamping load, each time removing and reapplying the sealant layer. The thickness of the interlayer gap width was then determined as the difference between the measured total stack thickness
Fig. 2 Vice used to clamp the aluminium-aluminium stacks with pre-set clamping forces

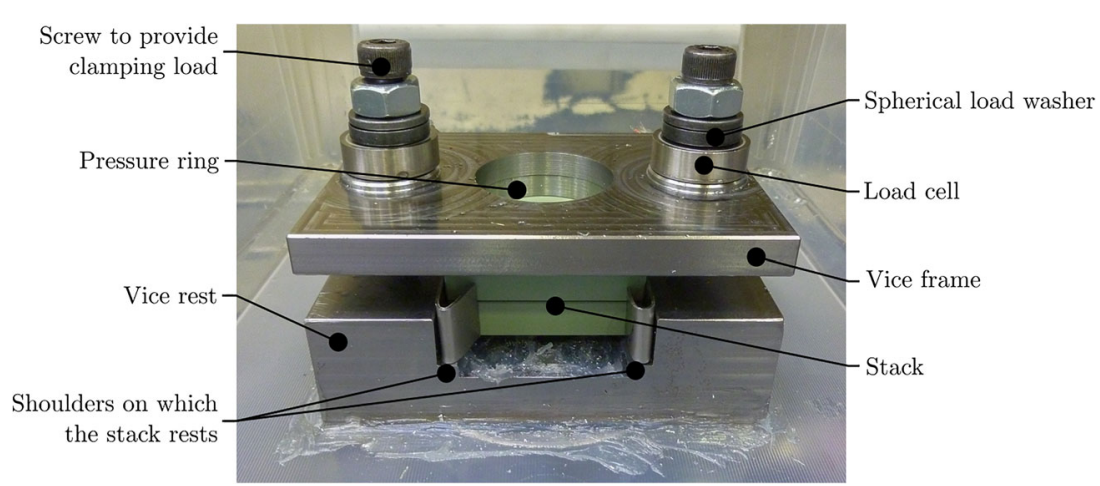


(i.e. including sealant layer) and the combined thickness of the two aluminium coupons (i.e. without sealant).

Figure 3 shows that an increase in clamping load brings about a noticeable reduction in the interlayer gap width, which follows the relationship as indicated by the equation in the figure (in which $\mathrm{x}$ represents the clamping force width and $y$ the interlayer gap width). This can be attributed to sealant movement within the interlayer as well as to some of the sealant being progressively squeezed out of the stack with increasing clamping force. In the case of the smallest clamping load, i.e. $100 \mathrm{~N}$, the gap width was around $0.3 \mathrm{~mm}$, which was reduced to $0.1 \mathrm{~mm}$ once the clamping load had been increased to $500 \mathrm{~N}$. When applying the maximum load of 3,000 N, the gap width was reduced to approximately zero, indicating that almost all of the sealant had been squeezed out from the gap.

In response to the above-mentioned findings, the drilling experiments were carried out using shims (i.e. thin metal strips serving as spacers) of thicknesses similar to the interlayer gap widths measured when applying clamping loads ranging from $100 \mathrm{~N}$ to $3000 \mathrm{~N}$. These shims served three purposes. Firstly, they made sure that the interlayer gap width could be set to the pre-defined values (see Table 1). Secondly, they ensured that no sealant was squeezed out from the interface during the drilling cycle in response to the thrust force, which would have resulted in a change of the interlayer gap width. Thirdly, the presence of shims prevented changes of the interlayer gap width as a result of variations in the amount of sealant applied.

\subsection{Drilling experiments}

Prior to clamping, a sufficiently large quantity of sealant was applied to the bottom plate of the stack to cover $80-90 \%$ of the plate's area, and two narrow metal shims were placed

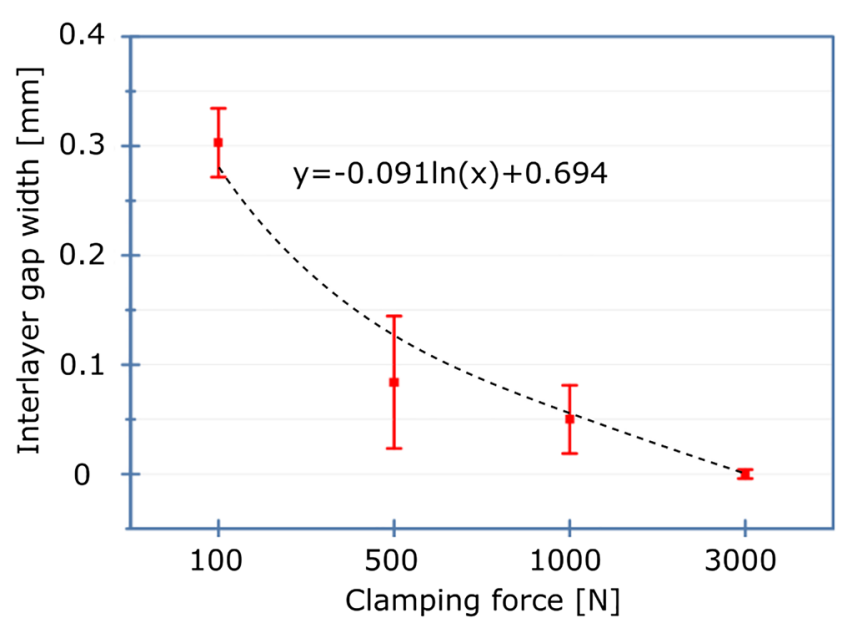

Fig. 3 Interlayer gap width of stack with sealant in relation to clamping force in between the two layers along two opposite edges of the stack. The stack was then placed inside the vice and the two screws connecting the vice's rest and frame were tightened to provide the total clamping load. One hole was then drilled at the centre of each coupon. The procedure was repeated using shims of different thicknesses to represent different interlayer gap widths. Two coupons were drilled for each pre-set interlayer gap.

\subsection{Burr height measurement}

After separation, the plates were washed in dichloromethane for around 60 seconds, which was sufficient to dissolve the sealant without having to apply any force to the part, e.g. wiping off the sealant, which could have potentially damaged the burr. It was decided to measure the height of the exit interlayer burr, i.e. the burr on the bottom surface of the top layer, which is believed to be the main contributor to the overall interlayer burr formation in stack drilling [5]. For each hole, two line scans using a contactless profilometer (Nanofocus $\mu \mathrm{m}$ scan) were carried out across the centre of the hole, at $0^{\circ}$ and $90^{\circ}$, thereby gathering data representing the borehole edges in four locations $\left(0^{\circ}, 90^{\circ}, 180^{\circ}\right.$ and $\left.270^{\circ}\right)$. The in-plane step size for these scans was $1 \mu \mathrm{m}$, and the resolution in the $\mathrm{z}$-axis (i.e. height) was $0.1 \mu \mathrm{m}$.

\section{Results and discussion}

\subsection{Interlayer burr formation process}

A visual assessment of the scan profiles obtained from the contactless profilometer revealed that a burr of traditional shape, i.e. a ridge of material adjacent to the borehole wall and protruding out from the surrounding material, was only present in a very few of the samples, most commonly those which were drilled without pre-set interlayer gap. For the majority of the samples, a much less uniform accumulation of material of varying height and width around the borehole was observed. In some cases, there was actually no accumulation of material whatsoever but removal of it around the borehole's edge. This removal was particularly noticed in the case of boreholes that were drilled with larger interlayer gaps, as shown in Fig. 4.

This of course made it very difficult to quantify any burr height, because as defined by ISO 13715 [13] and Ko and Dornfeld [14] a burr is a protruding feature, and in many cases the only protruding features identified were bulges of material in the vicinity of the borehole, but not directly located at the edge. The graph shown in Fig. 5 therefore refers to a "maximum surface level" rather than a burr height, in order to quantify the maximum height of any material accumulation around the borehole, but not 
Fig. 4 Typical borehole edge profiles when drilling stacks with different interlayer gap widths

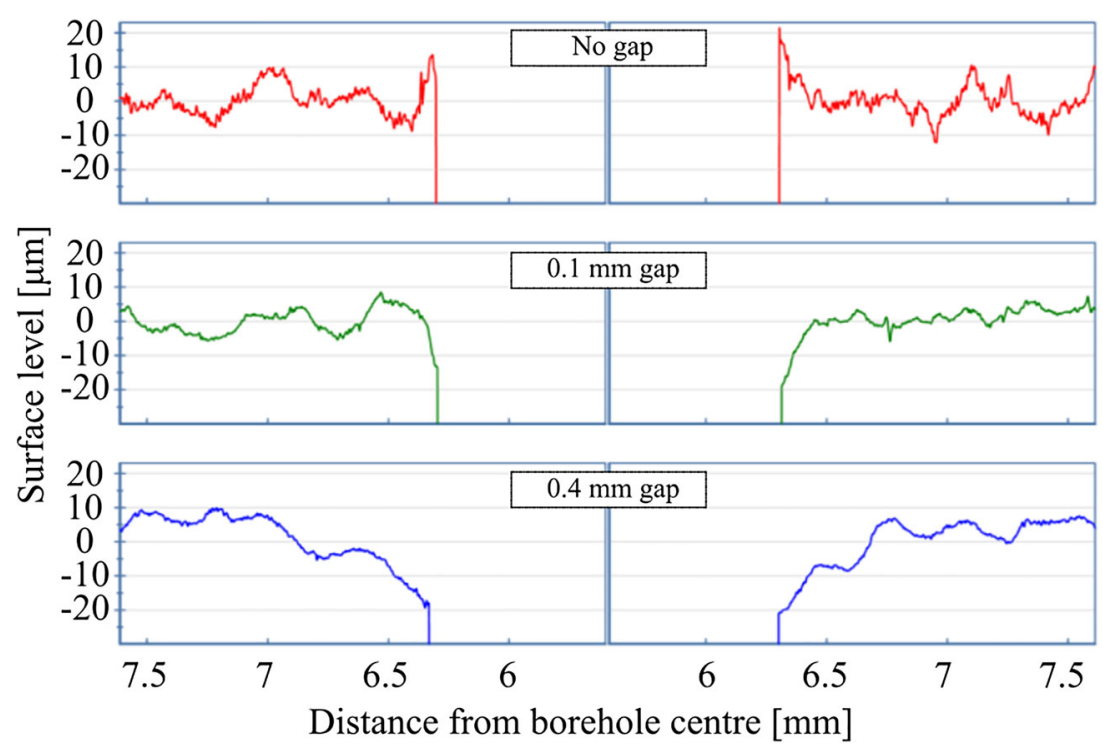

necessarily directly adjacent to it, and protruding out from the original workpiece surface. From the graph, it can clearly be seen how actual burrs were mainly measured around holes that were drilled without pre-set interlayer gap. In contrast, for the other experiments, the recorded maximum surface levels are quite constant and could well be a measure of the surface roughness of the shot-peened coupons as opposed to that of a burr formed on the borehole edge.

\subsection{Interlayer burr removal as a result of chip flow}

The separation of the plates after drilling often revealed significant swarf ingress in the interlayer gap, as can be seen in Fig. 6, which shows the coupon's bottom surface of the top layer around two boreholes drilled with an interlayer gap width of $0.3 \mathrm{~mm}$. A large amount of chips of varying size, ranging from tiny microscopic particles to large chip

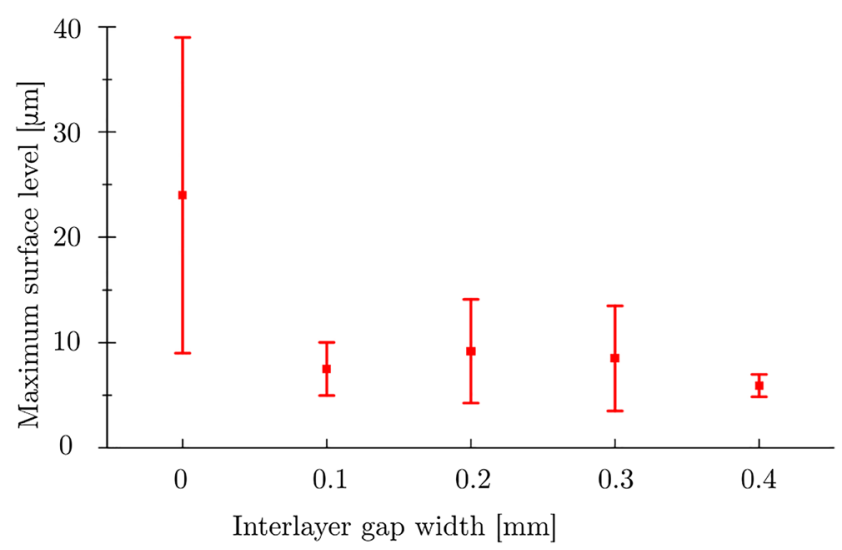

Fig. 5 Average maximum surface level with different pre-set interlayer gap widths segments, accumulated around the borehole on both the top and bottom plate. The largest portion of the swarf was typically located near the borehole edge, but in many cases smaller chip particles could also be found up to several millimetres away from the hole. Although it is believed that some spreading of the swarf did take place during the separation of the two plates-these were pulled apart in opposite directions- the extent of this cannot be quantified.

Based on a visual assessment of the boreholes using an optical microscope, an example of which is shown in Fig. 7, it appears that some abrasive action must have taken place around the material adjacent to the hole edges. From the images, it can be seen how the area adjacent to the borehole is covered with small scratch marks, which are believed to be the result of chips having penetrated into the interlayer gap and then being dragged around the hole by the rotating motion of the drill. In addition, substantial workpiece material abrasion at the borehole edge has occurred on the bottom surface of the upper layer, to the extent that the edge of the hole has the appearance of a chamfer or fillet. This implies the presence of considerable pressure between chips and workpiece material, and it is possible that the elevated process temperatures will have promoted this abrasive action as a result of thermal softening of the aluminium. It is thus believed that chip ingress and subsequent chip movement in the stack interface is the reason for the almost complete absence of any traditional burr in the case of pre-set interlayer gaps.

To gain a better understanding of the cause for this material abrasion around the borehole edges, the extent of interlayer damage at both the upper and lower interlayer surfaces was assessed in greater detail. Figure 8 shows the profile scans of one of the holes drilled. The two blue curves represent the edge profile of the exit of the borehole in 
Fig. 6 Accumulation of swarf around the borehole on the bottom surface of the top layer (interlayer gap width $0.3 \mathrm{~mm}$ )

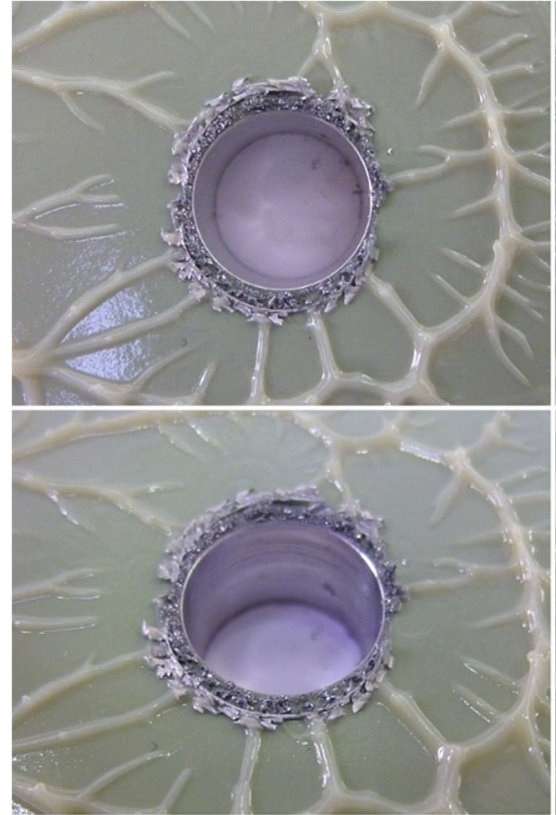

Hole 1

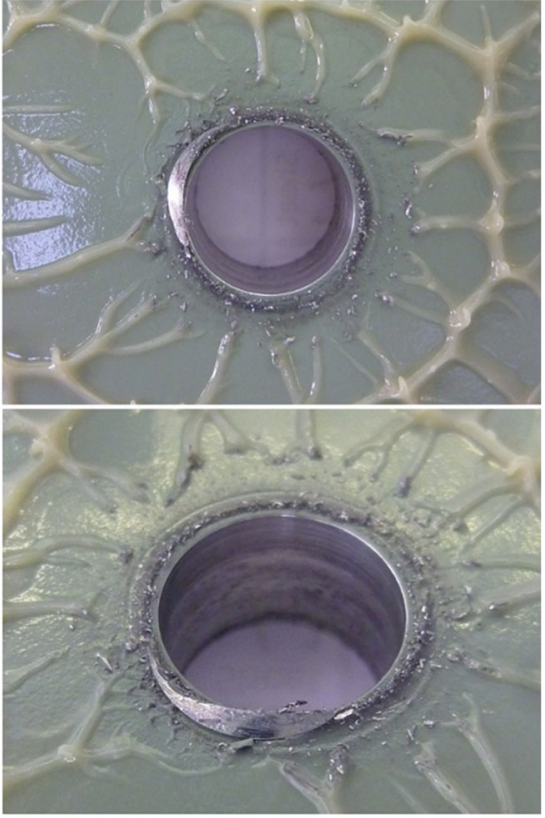

Hole 2 the bottom surface of the top layer, whereas the red curves represent the edge profile at the hole entrance in the top surface of the bottom layer. It can clearly be seen that the two profiles show significant differences, in as much as that the borehole edge in the bottom surface of the top plate has been completely rounded off. The radii along the edges of these holes were measured to be approximately $50-70 \mu \mathrm{m}$. The step in the blue profile, adjacent to the borehole edge, coincides with the clearly visible dark ring around the hole shown in Fig. 7. The borehole's edge in the bottom plate, despite some rounding, exhibits a noticeably smaller radius. Edge radii of approximately $10-15 \mu \mathrm{m}$ were measured. Similar differences were observed for all holes inspected, and these are believed to be a result of the direction of chip flow (indicated by the two dashed lines). With the tool moving into the stack, the chips flow in opposite direction to the feed, i.e. in this case upwards. As a consequence, they will flow over the borehole's edge in the bottom plate, thereby causing some abrasion and edge rounding. They will then flow further upwards, where some of them appear to get caught by the borehole edge on the underside of the top plate. Some will even manage to penetrate into the interlayer gap, where they are then being spun around by the rotating tool and, as a result, scrape over the interlayer surfaces, leaving behind scratch marks and, eventually, grinding away the burr that might have formed during tool exit from the top layer.

The cutting forces were consulted to find further evidence of the hypothesis postulated before. Typical examples of the recorded cutting forces during the experiments are shown in Fig. 9. The point where the drill reaches the stack interface, i.e. where it exits the top layer and is about to enter the bottom plate, can be clearly identified by the abrupt drop and then sudden rise in the thrust force, particularly in the case of a stack with a $0.3 \mathrm{~mm}$ interlayer gap. An increase in the torque signal dynamics when the tool is engaged in the bottom layer was also observed, in particular when drilling a stack with a $0.3 \mathrm{~mm}$ interlayer gap. This is believed to be in response to the chip ingress in the interlayer gap combined with, as a result of
Fig. 7 Surface damage of the material adjacent to the borehole for both interlayer surfaces (interlayer gap width $0.3 \mathrm{~mm}$ )

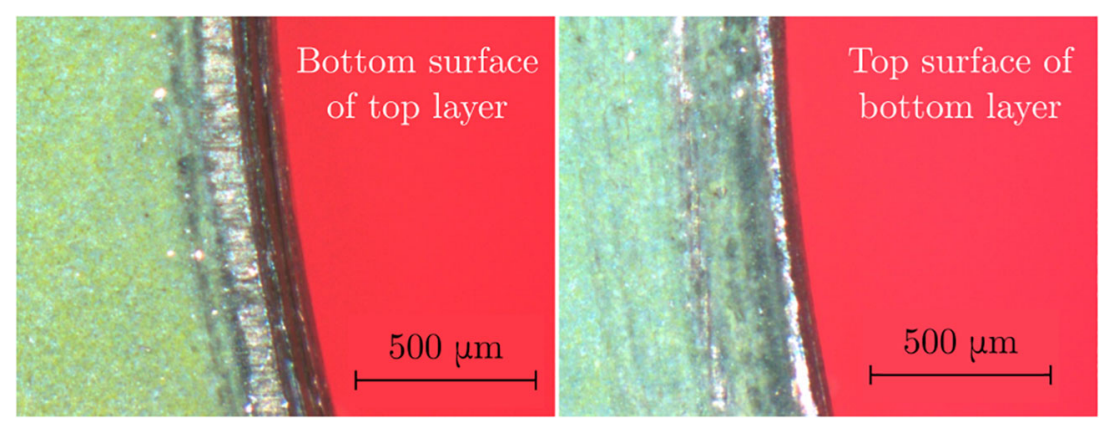




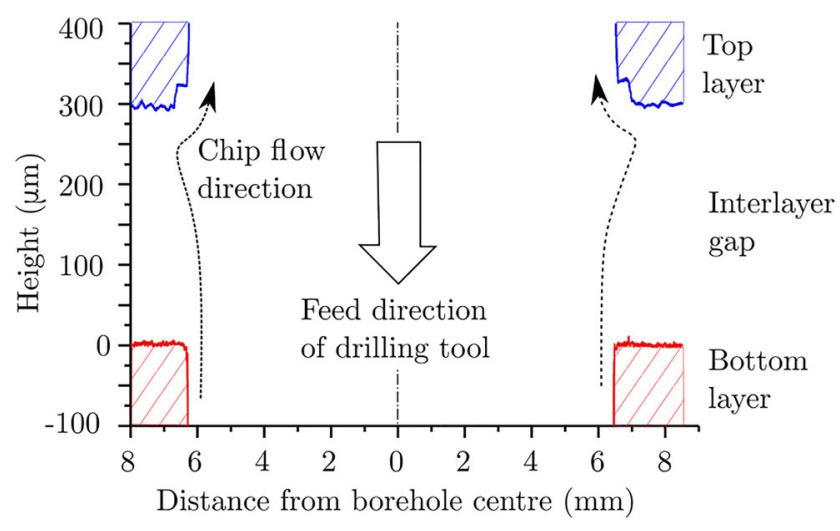

Fig. 8 Profiles of the interlayer surfaces in close proximity to the borehole (interlayer gap width $0.3 \mathrm{~mm}$ )

the rotating motion of the tool, the scraping of the chips over the interlayer surfaces. In contrast, a change in the torque amplitude could not be observed. Neither were there any changes with regards to the thrust force.

As a result of the drilling tool geometry and the cutting conditions utilised, both of which were in-line with current industrial practice, it was noticed that the chips generated were relatively thin, which would have made it easier for the chips to enter the interlayer gap. The thin chips also resulted in extensive chip nesting, which would have constrained the chip flow along the drill flutes, thereby increasing the chance of chips becoming entrapped in the interlayer gap and consequently abrading the newly formed burr.

\subsection{Impact of gap width on initial burr formation process in top layer}

To further validate the assumption made in the previous section, a second set of experiments was conducted, in which the drilling cycle was stopped once the tool's cutting edges got fully engaged with the second stack layer. The aim was to replicate the interval during which the exit interlayer burr is fully formed, i.e. drilling of top layer plus initial engagement with bottom layer, whilst preventing its potential removal when drilling through the bottom layer. This set of experiments was carried out using a stack without pre-set interlayer gap (i.e. gap width $0 \mathrm{~mm}$ ) and one with a $0.4 \mathrm{~mm}$ gap width. In addition to this, a further experiment was conducted using a single layer, to allow for the comparison between a constrained (i.e. stack interlayer) and unconstrained (i.e. single layer) situation.

In contrast to the burrs observed when drilling through an entire stack, those produced when stopping the drill's progression shortly after entering the bottom layer exhibited a more traditional profile. Moreover, the averages of the measured burr heights, see Fig. 10, indicate that a preset interlayer gap does indeed bring about a substantial
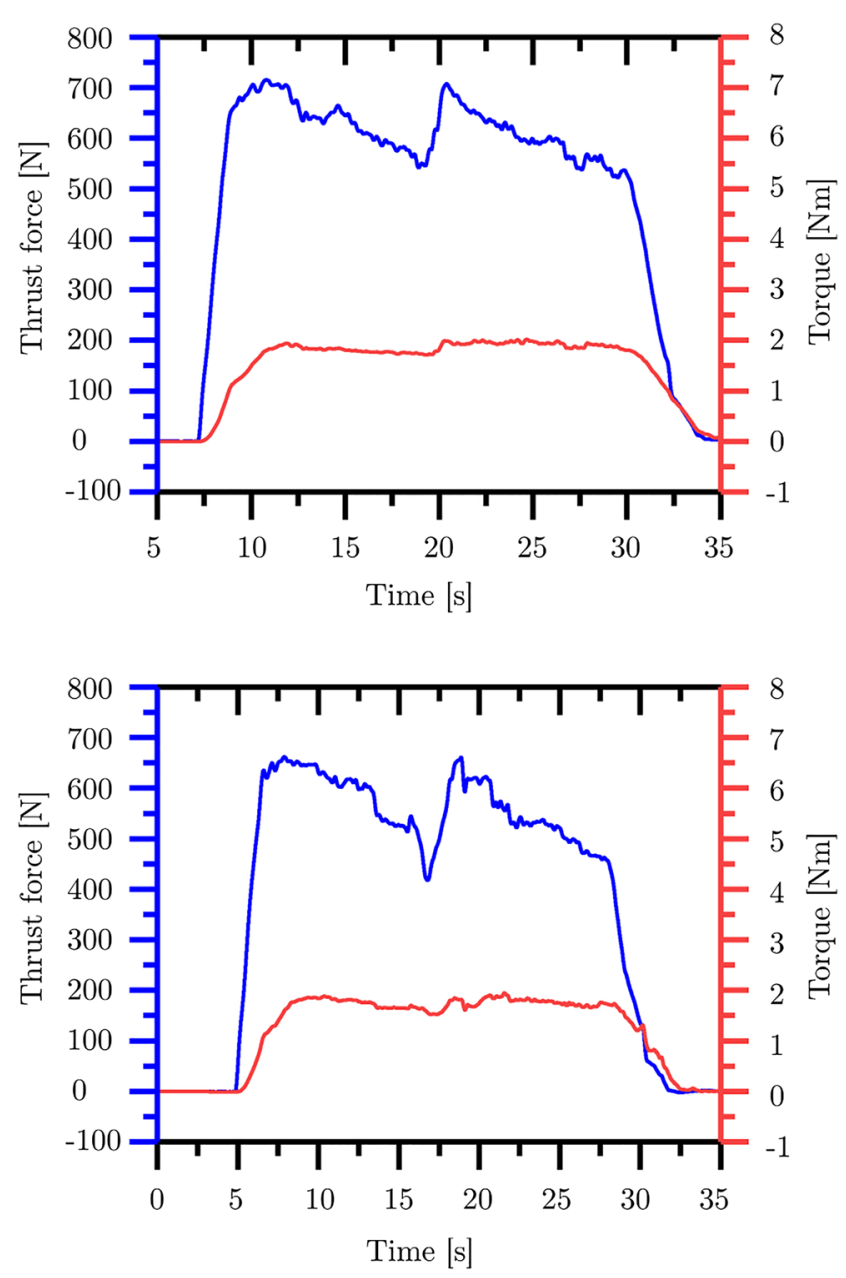

Fig. 9 Thrust force and torque recorded when drilling a stack with no interlayer gap (top) and with $0.3 \mathrm{~mm}$ interlayer gap width (bottom)

increase in interlayer burr height. Drilling a single layer, thereby creating the largest possible "interlayer" gap width, resulted in the largest exit burr. These observations confirm that during the previous experiments interlayer burrs were indeed formed and that these grew in height with the interlayer gap width, which is in agreement with previous

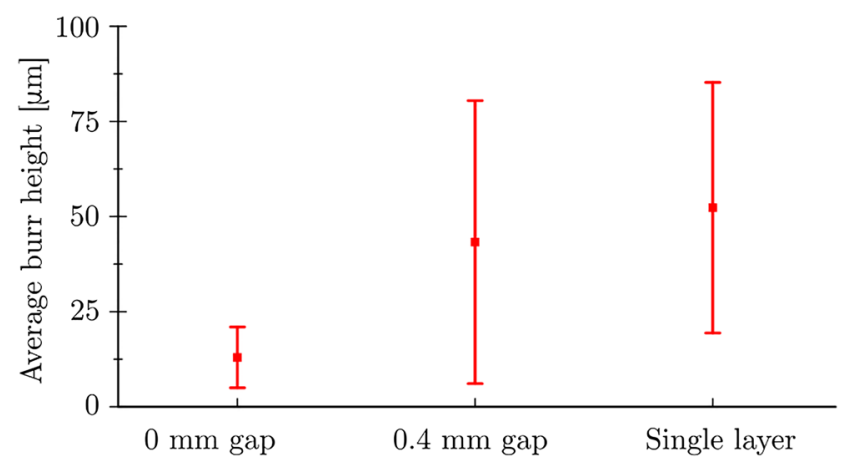

Fig. 10 Average burr height: drilling of top stack layer plus initial engagement with bottom stack layer at different interface conditions $(0 \mathrm{~mm}$ and $0.4 \mathrm{~mm}$ interlayer gap width), and drilling of single layer 
research [6, 9], but that these burrs were then eroded away by the upwards-travelling chips during the subsequent drilling of the bottom layer.

\section{Conclusions}

In this paper, the influence of interlayer gap width on interlayer burr formation when drilling aluminiumaluminium stacks with sealant applied at the interface has been experimentally investigated. The way in which the research was conducted, which differed from previous work in terms of how the interlayer gap was set and the workpiece size, revealed a phenomenon not identified before, which strongly affects the borehole quality at the interlayer gap.

Based on the observations made during the research, the following conclusions can be drawn:

- Pre-load clamping of stacks causes the sealant to be squeezed out from the stack interface. Increasing the clamping load results in an increase in the amount of sealant being squeezed out and, thus, in a reduction of the interlayer gap width. For the small-sized coupons used during this research a clamping force of $3,000 \mathrm{~N}$ was sufficient to reduce the interlayer gap width to zero, indicating that almost all sealant had been displaced.

- In drilling, the size of the burr strongly depends on the space available for the burr to protrude into. Introducing a pre-set interlayer gap to a stack results in a substantial increase in interlayer burr height as opposed to drilling a stack with no interlayer gap. Similarly, drilling single layers results in larger exit burrs being formed when compared to the interlayer burrs formed in stacks drilled with a pre-set interlayer gap.

- The presence of an interlayer gap can however be detrimental to burr formation, which is due to two phenomena: the sliding action of the upwards-travelling chips over the borehole edges, in combination with some of the chips entering the gap and being spun around by the rotating tool, thereby further eroding the borehole edge as well as the newly formed burr. This abrasion is considerable at the borehole edge of the upper layer (i.e. exit interlayer burr), but less pronounced at the edge of the bottom layer (i.e. entry interlayer burr). This is attributed to the fact that the former represents a greater obstacle to the chip flow and, hence, experiences a more intimate workpiece-chip contact.

- The abrasive action of the chips intensifies with an increase in the interlayer gap width, as this provides more space available for the chips to enter the interface and, thus, erode the burr. Hence, larger interlayer gap widths can result in the complete removal of the interlayer burr and potentially lead to a substantial rounding of the borehole edge. Excessive rounding as a result of a continuous and intensive chip flow across a wide interlayer gap could jeopardise the quality of the workpiece, as it affects the geometry of the borehole.

Acknowledgements This project was financially supported by Airbus UK (SP1702924), who also provided the workpiece material, sealant, coolant and cutting tools for the experimental work.

Open Access This article is distributed under the terms of the Creative Commons Attribution 4.0 International License (http:// creativecommons.org/licenses/by/4.0/), which permits unrestricted use, distribution, and reproduction in any medium, provided you give appropriate credit to the original author(s) and the source, provide a link to the Creative Commons license, and indicate if changes were made.

\section{References}

1. Cheng H, Li H, Zhang Y, Su KF, Bin J (2011) Efficient method of positioning error analysis for aeronautical thin-walled structures multi-state riveting. Int J Adv Manuf Technol 55:217233. https://doi.org/10.1007/s00170-010-3020-9

2. Shyha IS, Soo SL, Aspinwall DK, Bradley S, Perry R, Harden P, Dawson S (2011) Hole quality assessment following drilling of metallic-composite stacks. Int J Mach Tools Manuf 51:569-578. https://doi.org/10.1016/j.ijmachtools.2011.04.007

3. Liang X, Wu D, Gao Y, Chen K (2018) Investigation on the non-coaxiality in the drilling of carbon-fibre-reinforced plastic and aluminium stacks. Int $\mathrm{J}$ Mach Tools Manuf 125:1-10. https://doi.org/10.1016/j.ijmachtools.2017.11.001

4. Luo B, Li Y, Zhang K, Cheng H, Liu S (2015) A novel prediction model for thrust force and torque in drilling interface region of CFRP/Ti stacks. Int J Adv Manuf Technol 81:1497-1508. https://doi.org/10.1007/s00170-015-7294-9

5. Zhu Z, Guo K, Sun J, Li J, Liu Y, Zheng Y, Chen L (2018) Evaluation of novel tool geometries in dry drilling aluminium 2024-T351/titanium Ti6Al4V stack. J Mater Process Technol 259:270-281. https://doi.org/10.1016/j.jmatprotec.2018.04.044

6. Melkote SN, Newton TR, Hellstern C, Morehouse J, Turner S (2010) Interfacial burr formation in drilling of stacked aluminum sheets. In: Burrs - analysis, control and removal. pp 89-98

7. Tian W, Hu J, Liao W, Bu Y, Zhang L (2016) Formation of interlayer gap and control of interlayer burr in dry drilling of stacked aluminum alloy plates. Chinese J Aeronaut 29:283-291. https://doi.org/10.1016/j.cja.2015.11.002

8. Pande SS, Relekar HP (1986) Investigations on reducing burr formation in drilling. Int $\mathrm{J}$ Mach Tool Des Res 26:339-348. https://doi.org/10.1016/0020-7357(86)90010-7

9. Jie L (2013) The formation and effect of interlayer gap in dry drilling of stacked metal materials. Int $\mathbf{J}$ Adv Manuf Technol 69:1263-1272. https://doi.org/10.1007/s00170-013-5112-9

10. Li Y, Hu YX, Yao ZQ (2012) Modeling and analysis of the effect of preloaded pressing force on gap formation during the drilling of double-layered material. Appl Mech Mater 217-219:1541-1546. https://doi.org/10.4028/www.scientific.net/AMM.217-219.1541

11. Yin B, Wei T, WenHe L, Jian H, Xin S (2015) Investigation of correlation between interlayer gap and burr height in drilling of stacked Al-7475 materials. Proc Inst Mech Eng Part B J Eng Manuf 1-14:1-14. https://doi.org/10.1177/0954405415617671 
12. Gao Y, Wu D, Nan C, Ma X, Dong Y, Chen K (2015) The interlayer gap and non-coaxiality in stack drilling. Int $\mathrm{J}$ Mach Tools Manuf 99:68-76. https://doi.org/10.1016/j.ijmachtools.2015.09. 007

13. International Standards Organisation (2017) ISO 13715:2017(en) Technical product documentation - edges of undefined shape indication and dimensioning
14. Ko S-L, Dornfeld DA (1991) A study on burr formation mechanism. J Eng Mater Technol 113:75-87. https://doi.org/10.1115/1. 2903385

Publisher's note Springer Nature remains neutral with regard to jurisdictional claims in published maps and institutional affiliations. 